\title{
DIVISION ALGEBRAS AND FIBRATIONS OF SPHERES BY GREAT SPHERES
}

\author{
C. T. YANG
}

Dedicated to Professor Buchin Su on his 80 th birthday

Smooth fibrations of spheres by great spheres occur naturally in the study of the Blaschke conjecture. In fact, if $M$ is a Blaschke manifold, $m$ is a point of $M, T_{m} M$ is the tangent space of $M$ at $m$, $\exp _{m}: T_{m} M \rightarrow M$ is the exponential map at $m$, and $\operatorname{Cut}(m)$ is the cut locus of $m$ in $M$, then $\exp _{m}^{-1}(\operatorname{Cut}(m))$ is a sphere $S_{m}$ in $T_{m} M$ of center 0 , and $\exp _{m}: S_{m} \rightarrow \operatorname{Cut}(m)$ is a smooth great sphere fibration of the sphere $S_{m}$. For general information of the Blaschke conjecture, see [2].

If $\mathbf{K}$ is the real, complex, quaternionic or Cayley algebra, $n$ is the dimension of $\mathbf{K}$ as a euclidean space, which is $1,2,4$ or 8 , and $S^{2 n-1}$ is the unit $(2 n-1)$-sphere in the euclidean $2 n$-space $\mathbf{K} \times \mathbf{K}$, then there is a natural smooth great $(n-1)$-sphere fibration of $S^{2 n-1}$ such that any $(u, w),\left(u^{\prime}, w^{\prime}\right) \in$ $S^{2 n-1}$ belong to the same fibre iff either $w=w^{\prime}=0$ or $u w^{-1}=u^{\prime} w^{\prime-1}$. When $n>1$, this fibration, as well as isomorphic ones, is often referred as the Hopf fibration. Related to this result, Adams' theorem [1] says that a smooth fibration of $S^{2 n-1}$ by $(n-1)$-spheres can occur only when $n=1,2,4$ or 8 , and a classical theorem of Hurwitz [4] says that any division algebra $\mathbf{K}$, which possesses a norm such that for any $v, w \in \mathbf{K},|v w|=|v||w|$, must be the real, complex, quaternionic or Cayley algebra. If $n=1$ or 2 , then any $n$-dimensional division algebra is the real or complex algebra, and any fibration of $S^{2 n-1}$ by $(n-1)$-spheres is unique up to an isomorphism. Hence in these cases, the correspondence between $n$-dimensional division algebras and smooth great $(n-1)$-sphere fibrations of $S^{2 n-1}$ is trivial.

In this paper, we show that for $n=4$ or 8 , each $n$-dimensional division algebra $\mathbf{K}$ determines a smooth great $(n-1)$-sphere fibration of $S^{2 n-1}$, and every smooth great $(n-1)$-sphere fibration of $S^{2 n-1}$, up to an isomorphism, is determined by an $n$-dimensional division algebra $\mathbf{K}$. However, it is possible

Received December 23, 1981. The author is supported in part by the National Science Foundation. 
that two division algebras, not isomorphic to each other, may determine isomorphic smooth great $(n-1)$-sphere fibrations of $S^{2 n-1}$. Such an example can be found using division algebras constructed in Bruck [3].

We also show that any division algebra of dimension $>1$ contains the complex algebra as a subalgebra. Results of a subsequent paper of the author's joint work with Herman Gluck and Frank Warner will be used to show that any smooth great 3-sphere fibration of $S^{7}$ is isomorphic to the Hopf fibration, and hence any Blaschke manifold which has the integral cohomology ring of the quaternionic projective 2-space $\mathbf{H} P^{2}$ is homeomorphic to $\mathbf{H} P^{2}$.

The author wishes to express his gratitude to many colleagues of his for numerous dicussions, and especially to McKenzie $Y$. Wang for bringing Bruck's paper to his attention, and to Stephen S. Shatz for showing him an algebraic proof of the result that any division algebra of dimension $>1$ contains the complex algebra.

Throughout this paper, $\mathbf{R}$ denotes the real algebra, and $\mathbf{C}$ the complex algebra. Let $\mathbf{K}$ be the euclidean $n$-space, $n \geqslant 1$, which is often regarded as a vector space over $\mathbf{R}$. By a regular multiplication on $\mathbf{K}$, we mean a bilinear function

$$
m: \mathbf{K} \times \mathbf{K} \rightarrow \mathbf{K}
$$

such that for any $a, b \in \mathbf{K}$ with $a \neq 0$, each of

$$
m(v, a)=b, \quad m(a, w)=b
$$

has a unique solution in $\mathbf{K}$. $\mathbf{K}$ together with a regular multiplication on $\mathbf{K}$ is called a regular algebra which we also denote by $\mathbf{K}$. If $m$ is the only regular multiplication on $\mathbf{K}$ under our consideration, we often write $v w$ in place of $m(v, w)$. We note that a regular multiplication may not be associative, and a regular algebra may have no identity, and that a regular algebra may not have a norm such that the norm of a product is equal to the product of the norms. On the other hand, it can be shown that any 1-dimensional regular algebra must be $\mathbf{R}$, and that the dimension of any regular algebra is $1,2,4$ or 8 . A division algebra is defined to be a regular algebra having an identity. Notice that the real, complex, quaternionic and Cayley algebras are division algebras.

Let $\left\{e_{1}, \cdots, e_{n}\right\}$ be a basis of $\mathbf{K}$ as a vector space over $\mathbf{R}$. Then for any bilinear function $m: \mathbf{K} \times \mathbf{K} \rightarrow \mathbf{K}$, there are $n^{3}$ real numbers $a_{i j k}, i, j, k=$ $1, \cdots, n$, such that

$$
m\left(\sum_{i=1}^{n} v_{i} e_{i}, \sum_{k=1}^{n} w_{k} e_{k}\right)=\sum_{j=1}^{n}\left(\sum_{i, k=1}^{n} v_{i} a_{i j k} w_{k}\right) e_{j} .
$$

Hence regular multiplications are always smooth. 
Proposition 1. Any 1-dimensional regular algebra is the real algebra.

Proof. Let $\mathbf{K}$ be a 1-dimensional regular algebra, and let $a$ be an element of $\mathbf{K}$ different from the zero of $\mathbf{K}$. By definition, $a e=a$ for some $e \in \mathbf{K}$. $e$ is different from the zero of $\mathbf{K}$; otherwise, $a=a e=a(0 e)=0(a e)=0 e=e$, contradicting to our assumption.

Let $a=t e, t \in \mathbf{R}$. Then $t \neq 0$, and $t e=(t e) e=t e^{2}$ so that $e^{2}=e$. Hence $e$ is the identity of $\mathbf{K}$, and $\mathbf{K}$ can be naturally identified with $\mathbf{R}$ by setting $r e=r$ for all $r \in \mathbf{R}$.

Theorem 1. Any division algebra of dimension $>1$ contains a subalgebra isomorphic to the complex algebra.

Corollary 1. Any 2-dimensional division algebra is the complex algebra.

Let $\mathbf{K}$ be a division algebra of dimension $n>1$, and let $S^{2 n-1}$ be the unit $(n-1)$-sphere in $\mathbf{K}$. We may assume that the identity $e$ of $\mathbf{K}$ is contained in $S^{n-1}$; otherwise all we have to do is to use a new norm on $\mathbf{K}$ which is equal to $|e|^{-1}$ times the old one.

Lemma 1. The map $f: S^{n-1} \rightarrow S^{n-1}$ defined by $f(x)=x^{2} /\left|x^{2}\right|$ is of degree 2.

Proof. Let

$$
\phi: S^{n-1} \times S^{n-1} \rightarrow S^{n-1}
$$

be the map defined by

$$
\phi(x, y)=x y /|x y| \text {. }
$$

Notice that $\phi$ is well-defined and continuous, since $x y \in \mathbf{K}-\{0\}$ for any $x, y \in \mathbf{K}-\{0\}$.

Let $\Delta$ be the diagonal set in $S^{n-1} \times S^{n-1}$. Let $S^{n-1}$ be oriented, and let $S^{n-1} \times\{e\},\{e\} \times S^{n-1}$ and $\Delta$ be so oriented that the natural projection of each of them onto $S^{n-1}$ is orientation-preserving. Let

$$
\phi_{*}: H_{n-1}\left(S^{n-1} \times S^{n-1}\right) \rightarrow H_{n-1}\left(S^{n-1}\right)
$$

be the induced homomorphism of integral homology groups by $\phi$. Then

$$
\begin{gathered}
\phi_{*}\left[S^{n-1} \times\{e\}\right]=\left[S^{n-1}\right]=\phi_{*}\left[\{e\} \times S^{n-1}\right], \\
{[\Delta]=\left[S^{n-1} \times\{e\}\right]+\left[\{e\} \times S^{n-1}\right],}
\end{gathered}
$$

so that

$$
\phi_{*}[\Delta]=2\left[S^{n-1}\right]
$$

Since $\phi(x, x)=f(x)$ for any $x \in S^{n-1}$, our assertion follows.

Proof of Theorem 1. By Lemma 1, the map

$$
g: \mathbf{K} \rightarrow \mathbf{K}
$$


defined by $g(x)=x^{2}$ is onto. Therefore there is an element $i$ of $\mathbf{K}-\{0\}$ such that

$$
i^{2}=g(i)=-e \text {. }
$$

The linear 2-subspace of $\mathbf{K}$ having $\{e, i\}$ as a basis is clearly a subalgebra of $\mathbf{K}$ isomorphic to $\mathbf{C}$.

As mentioned earlier, Stephen S. Shatz has an algebraic proof of Lemma 1, and hence Theorem 1 can be proved algebraically.

Theorem 2. Let $\mathbf{K}$ be a regular algebra of dimension $n>1$, and let $S^{2 n-1}$ be the unit $(2 n-1)$-sphere in the euclidean $2 n$-space $\mathbf{K} \times \mathbf{K}$. Then $\mathbf{K}$ determines a smooth great $(n-1)$-sphere fibration of $S^{2 n-1}$ such that any $(u, w),\left(u^{\prime}, w^{\prime}\right) \in$ $S^{2 n-1}$ belong to the same fibre iff either $w=w^{\prime}=0$ or $u=v w$ and $u^{\prime}=v w^{\prime}$ for some $v \in \mathbf{K}$. Moreover, the fibrations determined by two isomorphic regular algebras are smoothly isomorphic.

Notice that if $\mathbf{K}$ is the complex, quaternionic or Cayley algebra, then the fibration determined by $\mathbf{K}$ is the Hopf fibration.

Proof. Let $\Sigma^{n}=\mathbf{K} \cup\{\infty\}$ be the one-point compactification of $\mathbf{K}$. Then $\Sigma^{n}$ can be made a smooth manifold as follows. For any $u \in \mathbf{K}-\{0\}$, we let

$$
\lambda_{u}: \Sigma^{n}-\{0\} \rightarrow \mathbf{K}
$$

be the homeomorphism such that $\lambda_{u}(\infty)=0$ and $v \lambda_{u}(v)=u$ for any $v \in \mathbf{K}-$ $\{0\}=\Sigma^{n}-\{0, \infty\}$. Since $\lambda_{u}: \mathbf{K}-\{0\} \rightarrow \mathbf{K}-\{0\}$ is a diffeomorphism, there is a smooth structure on $\Sigma^{n}$ such that the inclusion map of $\mathbf{K}$ into $\Sigma^{n}$ is a smooth imbedding, and $\lambda_{u}$ is a diffeomorphism for some $u \in \mathbf{K}-\{0\}$. The smooth structure on $\Sigma^{n}$ is independent of the choice of $u$. In fact, for any $u, u^{\prime} \in \mathbf{K}-\{0\}, u$ and $u^{\prime}$ can be joined by a smooth path in $\mathbf{K}-\{0\}$, and hence $\lambda_{u}, \lambda_{u^{\prime}}: \mathbf{K}-\{0\} \rightarrow \mathbf{K}-\{0\}$ are isotopic.

Let

$$
\pi: S^{2 n-1} \rightarrow \Sigma^{n}
$$

be the map such that $\pi(u, 0)=\infty$ for any $u \in S^{n-1}$, and $\pi(u, w) w=u$ for any $(u, w) \in S^{2 n-1}$ with $w \neq 0$. Since the multiplication on $\mathbf{K}$ is bilinear, it follows that $\pi^{-1} v$ is a great $(n-1)$-sphere in $S^{2 n-1}$ for any $v \in \Sigma^{n}$.

There is a smooth imbedding

$$
g_{0}: \mathbf{K} \times S^{n-1} \rightarrow S^{2 n-1}
$$

given by

$$
g_{0}(v, w)=\left(v w / \sqrt{|v w|^{2}+1}, w / \sqrt{|v w|^{2}+1}\right),
$$

and for any $v \in \mathbf{K}, \pi g_{0}\left(\{v\} \times S^{n-1}\right)=v$. Also there is a smooth imbedding

$$
g_{1}: S^{n-1} \times\left(\Sigma^{n}-\{0\}\right) \rightarrow S^{2 n-1}
$$


given by

$$
g_{1}(u, v)=\left(u / \sqrt{1+\left|\lambda_{u}(v)\right|^{2}}, \lambda_{u}(v) / \sqrt{1+\left|\lambda_{u}(v)\right|^{2}}\right),
$$

and for any $v \in \Sigma^{n}-\{0\}, \pi g_{1}\left(S^{n-1} \times\{v\}\right)=v$. Hence

$$
\pi: S^{2 n-1} \rightarrow \Sigma^{n}
$$

is a smooth great $(n-1)$-sphere fibration.

Let $K_{1}$ be a regular algebra isomorphic to $K$, and let

$$
\pi_{1}: S_{1}^{2 n-1} \rightarrow \Sigma^{n}
$$

be the smooth great $(n-1)$-sphere fibration determined by $\mathbf{K}_{1}$, where $S_{1}^{2 n-1}$ is the unit $(2 n-1)$-sphere in $\mathbf{K}_{1} \times \mathbf{K}_{1}$. Then $\pi_{1}: S_{1}^{2 n-1} \rightarrow \Sigma_{1}^{n}$ is smoothly isomorphic to $\pi: S^{2 n-1} \rightarrow \Sigma^{n}$. In fact, if $f: K_{1} \rightarrow \mathbf{K}$ is an isomorphism, then

$$
f \times f: \mathbf{K}_{1} \times \mathbf{K}_{1} \rightarrow \mathbf{K} \times \mathbf{K}
$$

defined by $(f \times f)\left(u_{1}, w_{1}\right)=\left(f u_{1}, f w_{1}\right)$ is a nonsingular linear map so that

$$
h: S_{1}^{2 n-1} \rightarrow S^{2 n-1}
$$

defined by $h\left(u_{1}, w_{1}\right)=\left(f u_{1}, f w_{1}\right) /\left|\left(f u_{1}, f w_{1}\right)\right|$ is a diffeomorphism. It is easy to see that $h$ maps fibres of $\pi_{1}: S_{1}^{2 n-1} \rightarrow \Sigma_{1}^{n}$ into fibres of $\pi: S^{2 n-1} \rightarrow \Sigma^{n}$. Hence the proof is completed.

As a consequence of Theorem 2 and Adams' theorem, we have

Corollary 2. The dimension of any regular algebra is 1,2,4 or 8 .

Let $G L(\mathbf{K})$ be the group of nonsingular linear maps of $\mathbf{K}$ into $\mathbf{K}$. Two regular multiplications $m$ and $m_{1}$ on $\mathbf{K}$ are said to be equivalent if there exist $\mu, \nu, \omega \in G L(\mathbf{K})$ such that $m_{1}(\nu \times \omega)=\mu m$, that means, the diagram

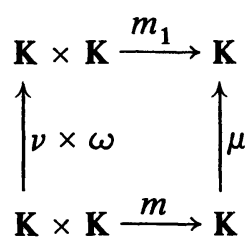

is commutative.

Proposition 2. Let $m$ and $m_{1}$ be equivalent regular multiplications on the euclidean $n$-space $\mathbf{K}$. Then the smooth great $(n-1)$-sphere fibrations of $S^{2 n-1}$ determined by the regular algebras $(\mathbf{K}, m)$ and $\left(\mathbf{K}, m_{1}\right)$ are smoothly isomorphic.

Proof. Let

$$
\pi: S^{2 n-1} \rightarrow \Sigma^{n}, \quad \pi_{1}: S^{2 n-1} \rightarrow \Sigma_{1}^{n}
$$

be the smooth great $(n-1)$-sphere fibrations determined by $(\mathbf{K}, m)$ and $\left(\mathbf{K}, m_{1}\right)$. Since $m$ and $m_{1}$ are equivalent, there are $\mu, \nu, \omega \in G L(\mathbf{K})$ such that 
$m_{1}(\nu \times \omega)=\mu m$. Then $\mu \times \omega: \mathbf{K} \times \mathbf{K} \rightarrow \mathbf{K} \times \mathbf{K}$ is a nonsingular linear map so that $h: S^{2 n-1} \rightarrow S^{2 n-1}$ defined by $h(u, w)=(\mu u, \omega w) /|(\mu u, \omega w)|$ is a diffeomorphism. It is easily seen that $h$ maps fibers of $\pi: S^{2 n-1} \rightarrow \Sigma^{n}$ into fibres of $\pi_{1}: S^{2 n-1} \rightarrow \Sigma_{1}^{n}$.

Proposition 3. On the euclidean $n$-space $\mathbf{K}$, any regular multiplication is equivalent to one having an identity.

Proof. Let $m$ be a regular multiplication on $\mathbf{K}$, and let

$$
\Phi, \Psi: \mathbf{K}-\{0\} \rightarrow G L(\mathbf{K})
$$

be the smooth maps such that

$$
\Phi(v) w=m(v, w), \quad \Psi(w) v=m(v, w) .
$$

Let $e \in \mathbf{K}-\{0\}$, let

$$
\mu, \nu, \omega: \mathbf{K} \rightarrow \mathbf{K}
$$

be the elements of $G L(K)$ given by

$$
\mu(u)=\Psi(e)^{-1} u, \quad \nu(v)=v, \quad \omega(w)=\Psi(e)^{-1} \Phi(e) w,
$$

and let $m^{\prime}$ be the regular multiplication on $\mathbf{K}$ such that

$$
m^{\prime}(v \times \omega)=\mu m \text {. }
$$

Then for any $v^{\prime}, w^{\prime} \in \mathbf{K}-\{0\}$,

$$
m^{\prime}\left(v^{\prime}, w^{\prime}\right)=\Psi(e)^{-1} m\left(v^{\prime}, \Phi(e)^{-1} \Psi(e) w^{\prime}\right)=\left\{\begin{array}{l}
\Psi(e)^{-1} \Phi\left(v^{\prime}\right) \Phi(e)^{-1} \Psi(e) w^{\prime} \\
\Psi(e)^{-1} \Psi\left(\Phi(e)^{-1} \Psi(e) w^{\prime}\right) v^{\prime}
\end{array}\right.
$$

Therefore

$$
m^{\prime}\left(e, w^{\prime}\right)=\Psi(e)^{-1} \Phi(e) \Phi(e)^{-1} \Psi(e) w^{\prime}=w^{\prime}
$$

so that

$$
e=m^{\prime}(e, e)=\Psi(e)^{-1} \Psi\left(\Phi(e)^{-1} \Psi(e) e\right) e .
$$

From the last equality, we infer that $\Phi(e)^{-1} \Psi(e) e=e$ and hence

$$
m^{\prime}\left(v^{\prime}, e\right)=\Psi(e)^{-1} \Psi\left(\Phi(e)^{-1} \Psi(e) e\right) v^{\prime}=v^{\prime} .
$$

As a consequence of Propositions 2 and 3, we have

Corollary 3. Any smooth great $(n-1)$-sphere fibration of $S^{2 n-1}$ determined by a regular algebra is smoothly isomorphic to one determined by a division algebra.

Now we are in a position to construct, from a given smooth great $(n-1)$ sphere fibration of $S^{2 n-1}$, an $n$-dimensional division algebra $\mathbf{K}$ such that the smooth great $(n-1)$-sphere fibration of $S^{2 n-1}$ determined by $\mathbf{K}$ is smoothly isomorphic to the given one. Since it is trivial for $n=1$ or 2 , in the following 
we assume that

$$
n=4 \text { or } 8 .
$$

Let $\mathbf{K}$ be the euclidean $n$-space, and $S^{n-1}$ the unit $(n-1)$-sphere in $\mathbf{K}$. Let $G L(K)$ be the group of all nonsingular linear maps of $\mathbf{K}$ into $\mathbf{K}$, and $S L(\mathbf{K})$ the subgroup of $G L(\mathbf{K})$ consisting of all the $g \in G L(\mathbf{K})$ with det $g=1$.

Let $L_{i}$ be a normed real vector $n$-space, and $S_{i}^{n-1}$ the unit $(n-1)$-sphere in $L_{i}, i=1,2$. A diffeomorphism $f: S_{1}^{n-1} \rightarrow S_{2}^{n-1}$ is called a linear diffeomorphism if there is a nonsingular linear map $g: L_{1} \rightarrow L_{2}$ such that for any $x \in S_{1}^{n-1}, f(x)=g(x) /|g(x)|$.

Lemma 2. Whenever $g \in G L(\mathbf{K})$, we have a linear diffeomorphism

$$
\bar{g}: S^{n-1} \rightarrow S^{n-1}
$$

defined by $g(x)=g(x) /|g(x)|$. Conversely, whenever $f: S^{n-1} \rightarrow S^{n-1}$ is a linear diffeomorphism, there is a unique $g \in G L(\mathbf{R})$ such that $\bar{g}=f$ and $\operatorname{det} g=$ \pm 1 , and $g^{\prime} g^{-1}$ is in the center of $G L(\mathbf{K})$ for any $g^{\prime} \in G L(\mathbf{K})$ with $\bar{g}^{\prime}=f$. Hence

$$
\overline{S L}(\mathbf{K})=\{\bar{g} \mid g \in S L(\mathbf{K})\}
$$

acts on $S^{n-1}$ as a smooth transformation group.

For any map $\alpha: S^{n-1} \rightarrow S L(\mathbf{K})$, we have a map $\bar{\alpha}: S^{n-1} \rightarrow \overline{S L}(\mathbf{K})$ defined by $\bar{\alpha}(v)=\overline{\alpha(v)}$, called the associated map of $\alpha$.

Lemma 3. Let $S_{i}^{n-1}$ be $S^{n-1}$ or a great $(n-1)$-sphere in $S^{2 n-1}, i=1,2$. Then any linear diffeomorphism $f: S_{1}^{n-1} \rightarrow S_{2}^{n-1}$ maps great circles into great circles, and any map f: $S_{1}^{n-1} \rightarrow S_{2}^{n-1}$ which maps great circles into great circles is a linear diffeomorphism.

Lemma 2 is quite obvious and Lemma 3 is a consequence of the well-known theorem in projective geometry that any map of a projective space of dimension $>1$ into itself which maps projective lines into projective lines is a projective transformation.

Let

$$
\pi: S^{2 n-1} \rightarrow \Sigma^{n}
$$

be a given smooth great $(n-1)$-sphere fibration of $S^{2 n-1}$. We first observe that $\Sigma^{n}$ is homeomorphic to the $n$-sphere. In fact, if $S^{n}$ is a great $n$-sphere in $S^{2 n-1}$ containing a fibre $F$, then $F$ is a great $(n-1)$-sphere in $S^{n}$, and $\Sigma^{n}$ is obtained from a closed hemisphere in $S^{n}$ with boundary $F$ by identifying $F$ to a single point.

Let $F_{0}$ and $F_{1}$ be two distinct fibres. Whenever $x$ is a point of $S^{2 n-1}-F_{i}, F_{i}$ and $x$ determine a great $n$-sphere in $S^{2 n-1}$. The closed hemisphere in this great $n$-sphere of boundary $F_{i}$ containing $x$ will be denoted by $F_{i} x$. 
Let

$$
h_{0}: S^{2 n-1}-F_{1} \rightarrow F_{0}, h_{1}: S^{2 n-1}-F_{0} \rightarrow F_{1}
$$

be the smooth maps such that for any $x \in S^{2 n-1}-F_{1-i}, h_{i}(x)$ is the point of intersection of $F_{1-i} x$ with $F_{i}, i=0,1$. Let

$$
x_{0}=\pi F_{0}, \quad x_{1}=\pi F_{1} .
$$

Then

$$
\begin{aligned}
& \pi \times h_{0}: S^{2 n-1}-F_{1} \rightarrow\left(\Sigma^{n}-\left\{x_{1}\right\}\right) \times F_{0}, \\
& h_{1} \times \pi: S^{2 n-1}-F_{0} \rightarrow F_{1} \times\left(\Sigma^{n}-\left\{x_{0}\right\}\right)
\end{aligned}
$$

are diffeomorphisms, which are local trivializations of the fibration over $\Sigma^{n}-\left\{x_{1}\right\}$ and $\Sigma^{n}-\left\{x_{0}\right\}$ respectively.

Let $S$ be the $(n-1)$-sphere of unit tangent vectors of $\Sigma^{n}$ at $x_{0}$ with respect to any preassigned Riemannian metric on $\Sigma^{n}$. Then for any $(v, w) \in S \times F_{0}$, there is a tangent vector $\tau(v, w)$ of $F_{1} w$ at $w$ such that

$$
d \pi(\tau(v, w))=v \text {. }
$$

Now we define a smooth map

$$
\xi: S \times F_{0} \rightarrow F_{1}
$$

as follows. Let $(v, w) \in S \times F_{0}$. Then there is a smooth map $f:[0,1] \rightarrow F_{1} w$ such that $f(t)=w$ iff $t=0$, and $f^{\prime}(0)=\tau(v, w)$. It is not hard to see that $\lim _{t \rightarrow 0} F_{0} f(t)$ exists and is a closed hemisphere of boundary $F_{0}$ with $\tau(v, w)$ as a tangent vector at $w . \xi(v, w)$ is defined to be the point of intersection of $\lim _{t \rightarrow 0} F_{0} f(t)$ with $F_{1}$.

The following lemma plays a key role in our paper.

Lemma 4. For any $v \in S, w \rightarrow \xi(v, w)$ is a linear diffeomorphism of $F_{0}$ onto $F_{1}$, and for any $w \in F_{0}, v \rightarrow \xi(v, w)$ is a linear diffeomorphism of $S$ onto $F_{1}$.

Proof. Let $v \in S$ and let $f:[0,1] \rightarrow \Sigma^{n}-\left\{x_{1}\right\}$ be a smooth map such that $f(t)=x_{0}$ iff $t=0$, and $f^{\prime}(0)=v$. Then for any $w \in F_{0}$, we have a smooth map $f_{w}:[0,1] \rightarrow F_{1} w$ such that $\pi f_{w}=f$. Clearly $f_{w}(t)=w$ iff $t=0$, and $f_{w}^{\prime}(0)=$ $\tau(v, w)$. Moreover,

$$
\xi(v, w)=\lim _{t \rightarrow 0} h_{1} f_{w}(t) .
$$

Let $C$ be a great circle in $F_{0}$. Then for any $t \in(0,1], C_{t}=\left\{f_{w}(t) \mid w \in C\right\}$ is the intersection of $\pi^{-1} f(t)$ with the great $(n+1)$-sphere in $S^{2 n-1}$ determined by $F_{1}$ and $C$, so that it is a great circle in $\pi^{-1} f(t)$. Therefore $h_{1}\left(C_{t}\right)$, which is the intersection of $F_{1}$ with the great $(n+1)$-sphere in $S^{2 n-1}$ determined by $F_{0}$ and $C_{t}$, is a great circle in $F_{1}$. Hence $\xi(v, C)=\lim _{t \rightarrow 0} h_{1}\left(C_{t}\right)$ is a great circle in $F_{1}$. From this result and Lemma 3 we conclude that $w \rightarrow \xi(v, w)$ is a linear diffeomorphism of $F_{0}$ onto $F_{1}$. 
Let $w \in F_{0}$. For any great circle $C$ in $S$ we have a great $(n+1)$-sphere $S^{n+1}$ in $S^{2 n-1}$ containing $F_{0}$ such that for any $v \in C, \tau(v, w)$ is a tangent vector of $S^{n+1}$ at $w$. It can be seen that $\xi(C, w)$ is the intersection of $F_{1}$ and $S^{n+1}$ so that it is a great circle in $F_{1}$. Hence by Lemma $3, v \rightarrow \xi(v, w)$ is a linear diffeomorphism of $S$ onto $F_{1}$.

Since $\Sigma^{n}$ is 1-connected, we may assume that $\pi: S^{2 n-1} \rightarrow \Sigma^{n}$ is oriented. Then for any $v \in S, w \rightarrow \xi(v, w)$ is an orientation-preserving linear diffeomorphism of $F_{0}$ onto $F_{1}$. We let $S$ be so oriented that for any $w \in F_{0}, v \rightarrow \xi(v, w)$ is also an orientation-preserving linear diffeomorphism of $S$ onto $F_{1}$.

Let $S^{n-1}$ be naturally oriented, and let us identify $F_{0}, F_{1}$ and $S$ with $S^{n-1}$ by orientation-preserving linear diffeomorphisms. Then $\xi: S \times F_{0} \rightarrow F_{1}$ becomes a smooth map

$$
\xi: S^{n-1} \times S^{n-1} \rightarrow S^{n-1}
$$

such that for some smooth maps

$$
\phi, \psi: S^{n-1} \rightarrow S L(\mathbf{K}),
$$

we have

$$
\xi(v, w)=\bar{\phi}(v) w=\bar{\psi}(w) v,
$$

where $\bar{\phi}, \bar{\psi}: S^{n-1} \rightarrow \overline{S L}(\mathbf{K})$ are the associated maps of $\phi$ and $\psi$.

The following result can be proved in the same way as Proposition 3.

Lemma 5. For any $e \in S^{n-1}$, we let

$$
\mu_{e}=\psi(e)^{-1}, \quad \nu_{e}=\text { identity, } \omega_{e}=\psi(e)^{-1} \phi(e),
$$

let

$$
\phi_{e}, \psi_{e}: S^{n} \rightarrow S L(\mathbf{K})
$$

be the smooth maps defined by

$$
\begin{aligned}
& \phi_{e}(v)=\mu_{e} \phi\left(\nu_{e}^{-1} v\right) \omega_{e}^{-1} \\
& \psi_{e}(w)=\mu_{e} \psi\left(\omega_{e}^{-1} w\right) \nu_{e}^{-1}
\end{aligned}
$$

and let

$$
\xi_{e}: S^{n-1} \times S^{n-1} \rightarrow S^{n-1}
$$

be the smooth map defined by

$$
\xi_{e}(v, w)=\bar{\mu}_{e} \xi\left(\bar{\nu}_{e}^{-1} v, \bar{\omega}_{e}^{-1} w\right) .
$$

Then

$$
\begin{gathered}
\phi_{e}(e)=\psi_{e}(e)=\text { identity, } \\
\xi_{e}(v, w)=\bar{\phi}_{e}(v) w=\bar{\psi}_{e}(w) v,
\end{gathered}
$$

where $\bar{\phi}_{e}, \bar{\psi}_{e}: S^{n-1} \rightarrow \overline{S L}(\mathbf{K})$ are the associated maps of $\phi_{e}$ and $\psi_{e}$. 
Lemma 6. $\mathbf{K}$ can be made a division algebra with identity e such that for any $v, w \in S^{n-1}$,

$$
\xi_{e}(v, w)=v w /|v w| .
$$

The following results are needed in the proof of Lemma 6.

Sublemma 1. Let $U$ be a nonnull open subset of $\mathbf{R}$, and let $\nu, \omega: U \rightarrow \mathbf{R}$ and $\alpha: \mathbf{R} \rightarrow \mathbf{R}$ be smooth maps such that

$$
\alpha(r)>0
$$

for any $r \in \mathbf{R}$, and

$$
\alpha(r)=\frac{1+\nu(s) r}{1+\omega(s) r}
$$

for any $r \in \mathbf{R}$ and $s \in U$. Then

$$
\nu=\omega, \quad \alpha=1
$$

Proof. By hypothesis,

$$
\alpha(r)(1+\omega(s) r)=1+\nu(s) r .
$$

Partially differentiating the equality with respect to $s$, we obtain

$$
\alpha(r) \omega^{\prime}(s) r=\nu^{\prime}(s) r .
$$

Therefore

$$
\alpha(r) \omega^{\prime}(s)=\nu^{\prime}(s) .
$$

If $\omega^{\prime}(s) \neq 0$, then $\alpha(r)=\nu^{\prime}(s) / \omega^{\prime}(s)$ which is independent of the choice of $r$. Therefore $\alpha(r)=\alpha(0)=1$ and hence

$$
\alpha=1 \text {. }
$$

If $\omega^{\prime}(s) \equiv 0$, then $\nu^{\prime}(s) \equiv 0$. Therefore there are $\nu, \omega \in \mathbf{R}$ such that

$$
\alpha(r)=\frac{1+\nu r}{1+\omega r} .
$$

Since $\alpha(r)>0$ for all $r \in \mathbf{R}$, it follows that $\nu=\omega$. Hence $\alpha(r)=1$ for all $r \in \mathbf{R}$ or

$$
\alpha=1 \text {. }
$$

Sublemma 2. Let $\lambda_{1}, \lambda_{2}, \mu_{1}, \mu_{2}, \alpha: \mathbf{R} \rightarrow \mathbf{R}$ be smooth maps such that

$$
\alpha(r)>0
$$

for any $r \in \mathbf{R}$, and

$$
\alpha(r)=\frac{1+\lambda_{1}(s) r+\lambda_{2}(s) r^{2}}{1+\mu_{1}(s) r+\mu_{2}(s) r^{2}}
$$

for any $r, s \in \mathbf{R}$. Then either $\lambda_{1}, \lambda_{2}, \mu_{1}, \mu_{2}$ are constant maps or $\alpha=1$. 
Proof. By hypothesis,

$$
\alpha(r)\left(1+\mu_{1}(s) r+\mu_{2}(s) r^{2}\right)=1+\lambda_{1}(s) r+\lambda_{2}(s) r^{2} .
$$

Partially differentiating the equality with respect to $s$, we obtain

$$
\alpha(r)\left(\mu_{1}^{\prime}(s) r+\mu_{2}^{\prime}(s) r^{2}\right)=\lambda_{1}^{\prime}(s) r+\lambda_{2}^{\prime}(s) r^{2} .
$$

Therefore

$$
\alpha(r)\left(\mu_{1}^{\prime}(s)+\mu_{2}^{\prime}(s) r\right)=\lambda_{1}^{\prime}(s)+\lambda_{2}^{\prime}(s) r .
$$

Assume first that $\mu_{1}^{\prime}(s) \equiv 0$. Then

$$
\lambda_{1}^{\prime}(s)=\alpha(0) \mu_{2}^{\prime}(s) 0 \equiv 0,
$$

so that

$$
\alpha(r) \mu_{2}^{\prime}(s)=\lambda_{2}^{\prime}(s) .
$$

If $\mu_{2}^{\prime}(s) \equiv 0$, then $\lambda_{2}^{\prime}(s) \equiv 0$. Hence $\lambda_{1}, \lambda_{2}, \mu_{1}, \mu_{2}$ are constant maps. If $\mu_{2}^{\prime}(s) \neq 0$, then there is a nonnull open subset $U$ of $\mathbf{R}$ such that for any $s \in U$, $\mu_{2}^{\prime}(s) \neq 0$. Therefore for any $r \in \mathbf{R}$ and $s \in U, \alpha(r)=\lambda_{2}^{\prime}(s) / \mu_{2}^{\prime}(s)$ which is independent of the choice of $r$. Hence $\alpha(r)=\alpha(0)=1$ or $\alpha=1$.

Assume next that $\mu_{1}^{\prime}(s) \neq 0$. Then there is a nonnull open subset $U$ of $\mathbf{R}$ such that $\mu_{1}^{\prime}(s) \neq 0$ and

$$
\lambda_{1}^{\prime}(s)=\alpha(0) \mu_{1}^{\prime}(s)=\mu_{1}^{\prime}(s)
$$

for any $s \in U$. Therefore for any $r \in \mathbf{R}$ and $s \in U$,

$$
\alpha(r)=\frac{1\left(\lambda_{2}^{\prime}(s) / \mu_{1}^{\prime}(s)\right) r}{1+\left(\mu_{2}^{\prime}(s) / \mu_{1}^{\prime}(s)\right) r} .
$$

Hence by Sublemma 1,

$$
\alpha=1 \text {. }
$$

Proof of Lemma 6. In this proof, we drop the subscript $e$ from $\xi_{e}, \phi_{e}, \psi_{e}$ so that $\xi, \phi, \psi$ are actually $\xi_{e}, \phi_{e}, \psi_{e}$ of Lemma 5 .

Let

$$
\Phi, \Psi: \mathbf{K}-\{0\} \rightarrow G L(\mathbf{K})
$$

be the maps such that for any $v, w \in \mathbf{K}-\{0\}$,

$$
\Phi(v)=\frac{|v|}{|\phi(v /|v|) e|} \phi(v /|v|), \quad \Psi(w)=\frac{|w|}{|\psi(w /|w|) e|} \psi(w /|w|) .
$$

Then for any $v, w \in \mathbf{K}-\{0\}$,

$$
\begin{gathered}
\Phi(v) e=v=\Psi(e) v, \quad \Psi(w) e=w=\Phi(e) w \\
\Phi(v) w /|\Phi(v) w|=\Psi(w) v /|\Psi(w) v| .
\end{gathered}
$$


If we are able to show that for any $v, w \in \mathbf{K}-\{0\}$,

$$
\Phi(v) w=\Psi(w) v
$$

then $\mathbf{K}$ can be made a division algebra such that for any $v, w \in \mathbf{K}-\{0\}$, $v w=\Phi(v) w=\Psi(w) v$ so that for any $v, w \in S^{n-1}, \xi(v, w)=v w /|v w|$.

In the following, we let $v$ and $w$ be two fixed elements of $\mathbf{K}-\{0\}$. If $v=r e$ for some $r \in \mathbf{R}$, then

$$
\Phi(v) w=r \Phi(e) w=r \Psi(w) e=\Psi(w) v
$$

If $w=r e$ for some $r \in \mathbf{R}$, then $\Phi(v) w=r \Phi(v) e=r \Psi(e) v=\Psi(w) v$. Hence we may assume that $v, w \notin \mathbf{R} e$. Let $\gamma$ be the real number such that

$$
\Phi(v) w=\gamma \Psi(w) v
$$

We claim that $\gamma=1$.

Assume first that $e, v, w$ are not linearly independent. Then for some $t, t^{\prime} \in \mathbf{R}$,

$$
w=t e+t^{\prime} v, t^{\prime} \neq 0
$$

Let $\left\{e_{1}, \cdots, e_{n}\right\}$ be a basis of $\mathbf{K}$ such that

$$
e_{1}=e, e_{2}=v \text {, }
$$

and let $\gamma_{1}, \cdots, \gamma_{n} \in \mathbf{R}$ be such that

$$
\Psi\left(e_{2}\right) e_{2}=\gamma_{1} e_{1}+\cdots+\gamma_{n} e_{n} .
$$

If $\gamma_{1}=\gamma_{3}=\gamma_{4}=\cdots=\gamma_{n}=0$, then

$$
\Psi\left(e_{2}\right)\left(e_{2}-\gamma_{2} e_{1}\right)=\Psi\left(e_{2}\right) e_{2}-\gamma_{2} \Psi\left(e_{2}\right) e_{1}=\gamma_{2} e_{2}-\gamma_{2} e_{2}=0,
$$

which is impossible. Therefore $\gamma_{k} \neq 0$ for some $k \neq 2$. We may assume that

$$
\gamma_{1} \neq 0
$$

In fact, if $\gamma_{1}=0$, then $\gamma_{k} \neq 0$ for some $k>2$, so that by replacing $e_{k}$ by $e_{k}+e_{1}$ we obtain a new $\gamma_{1}$ different from 0 .

For any $r, s \in \mathbf{R}$, there are smooth real valued functions

$$
\alpha=\alpha(r), \quad \beta=\beta(s)
$$

such that

$$
\Phi\left(e_{1}+r e_{2}\right) e_{2}=\alpha \Psi\left(e_{2}\right)\left(e_{1}+r e_{2}\right), \quad \Psi\left(e_{1}+s e_{2}\right) e_{2}=\beta \Phi\left(e_{2}\right)\left(e_{1}+s e_{2}\right) .
$$

Clearly $\alpha(0)=1$ and $\alpha(r) \neq 0$ for all $r \in \mathbf{R}$. Hence $\alpha(r)>0$ for all $r \in \mathbf{R}$. Similarly $\beta(0)=1$ and $\beta(s)>0$ for all $s \in \mathbf{R}$. Now

$$
\begin{aligned}
\Phi\left(e_{1}+r e_{2}\right)\left(e_{1}+s e_{2}\right) & =\Phi\left(e_{1}+r e_{2}\right) e_{1}+s \Phi\left(e_{1}+r e_{2}\right) e_{2} \\
& =e_{1}+r e_{2}+s \alpha \Psi\left(e_{2}\right)\left(e_{1}+r e_{2}\right) \\
& =e_{1}+r e_{2}+s \alpha e_{2}+r s \alpha\left(\gamma_{1} e_{1}+\cdots+\gamma_{n} e_{n}\right) . \\
\Psi\left(e_{1}+s e_{2}\right)\left(e_{1}+r e_{2}\right) & =e_{1}+r \beta e_{2}+s e_{2}+r s \beta\left(\gamma_{1} e_{1}+\cdots+\gamma_{n} e_{n}\right) .
\end{aligned}
$$


Since the coefficients of $e_{1}, \cdots, e_{n}$ in $\Phi\left(e_{1}+r e_{2}\right)\left(e_{1}+s e_{2}\right)$ and those in $\Psi\left(e_{1}+s e_{2}\right)\left(e_{1}+r e_{2}\right)$ are proportional, we infer that

$$
\frac{1+r s \alpha \gamma_{1}}{1+r s \beta \gamma \gamma_{1}}=\frac{r+s \alpha+r s \alpha \gamma_{2}}{r \beta+s+r s \beta \gamma \gamma_{2}}=\frac{\alpha \gamma_{k}}{\beta \gamma \gamma_{k}}, \quad k>2 .
$$

Therefore

$$
\alpha=\frac{1+\left((\beta-1) / s+\beta \gamma \gamma_{2}\right) r-\left(\beta \gamma \gamma_{1}\right) r^{2}}{1+\left(\gamma_{2}+s \gamma_{1}-s \beta \gamma \gamma_{1}\right) r-\left(\beta \gamma_{1}\right) r^{2}}
$$

By Sublemma 2, either $\alpha=1$ or

$$
\left((\beta-1) / s+\beta \gamma \gamma_{2}\right)^{\prime}=\left(\beta \gamma \gamma_{1}\right)^{\prime}=\left(\gamma_{2}+s \gamma_{1}-s \beta \gamma \gamma_{1}\right)^{\prime}=\left(\beta \gamma_{1}\right)^{\prime}=0 .
$$

In the first case,

$$
(\beta-1) / s+\beta \gamma \gamma_{2}=\gamma_{2}+s \gamma_{1}-s \beta \gamma \gamma_{1}, \quad \beta \gamma \gamma_{1}=\beta \gamma_{1} .
$$

Since $\beta \gamma_{1} \neq 0$, it follows from the second equality that $\gamma=1$. Then the first equality becomes

$$
(\beta-1)\left(1 / s+\gamma_{2}+s \gamma_{1}\right)=0 .
$$

Therefore $\beta-1=0$ or $\beta=1$. In the second case, $\beta^{\prime}(s)=0$ so that $\beta(s)=$ $\beta(0)=1$. Then

$$
0=\left(\gamma_{2}+s \gamma_{1}-s \beta \gamma \gamma_{1}\right)^{\prime}=\gamma_{1}(1-\gamma)
$$

so that $\gamma=1$. Therefore $\alpha=1$. Hence we always have

$$
\alpha=1, \beta=1, \gamma=1 \text {. }
$$

Since $v=e_{2}$ and $w=t e_{1}+t^{\prime} e_{2}$, it follows that when $t=0$,

$$
\Phi(v) w=t^{\prime} \Phi\left(e_{2}\right) e_{2}=t^{\prime} \Psi\left(e_{2}\right) e_{2}=\Psi(w) v,
$$

and when $t \neq 0$,

$$
\Phi(v) w=t \Phi\left(e_{2}\right)\left(e_{1}+\left(t^{\prime} / t\right) e_{2}\right)=t \Psi\left(e_{1}+\left(t^{\prime} / t\right) e_{2}\right) e_{2}=\Psi(w) v
$$

Assume now that $e, v, w$ are linearly independent. Then there is a basis $\left\{e_{1}, \cdots, e_{n}\right\}$ of $\mathbf{K}$ such that

$$
e_{1}=e, e_{2}=v, e_{3}=w .
$$

Let $\gamma_{1}, \cdots, \gamma_{n} \in \mathbf{R}$ be such that

$$
\Psi\left(e_{3}\right) e_{2}=\gamma_{1} e_{1}+\cdots+\gamma_{n} e_{n}
$$

Then

$$
\begin{aligned}
& \Phi\left(e_{1}+r e_{2}\right)\left(e_{1}+s e_{3}\right)=e_{1}+r e_{2}+s \alpha e_{3}+r s \alpha\left(\gamma_{1} e_{1}+\cdots+\gamma_{n} e_{n}\right), \\
& \Psi\left(e_{1}+s e_{3}\right)\left(e_{1}+r e_{2}\right)=e_{1}+r \beta e_{2}+s e_{3}+r s \beta \gamma\left(\gamma_{1} e_{1}+\cdots+\gamma_{n} e_{n}\right) .
\end{aligned}
$$


Therefore

$$
\frac{1+r s \alpha \gamma_{1}}{1+r s \beta \gamma \gamma_{1}}=\frac{r+r s \alpha \gamma_{2}}{r \beta+r s \beta \gamma \gamma_{2}}=\frac{s \alpha+r s \alpha \gamma_{3}}{s+r s \beta \gamma \gamma_{3}}=\frac{\alpha \gamma_{k}}{\beta \gamma \gamma_{k}}, \quad k>3 .
$$

We may assume that one of $\gamma_{1}, \gamma_{2}, \gamma_{3}$ is not 0 . In fact, if $\gamma_{1}=\gamma_{2}=\gamma_{3}=0$, then for some $k>0, \gamma_{k} \neq 0$. By replacing $e_{k}$ by $e_{k}+e_{1}$ we obtain a new $\gamma_{1}$ different from 0 .

If either $\gamma_{1}$ or $\gamma_{3}$ is not 0 , from

$$
\frac{1+r s \alpha \gamma_{1}}{1+r s \beta \gamma \gamma_{1}}=\frac{s \alpha+r s \alpha \gamma_{3}}{s+r s \beta \gamma \gamma_{3}}
$$

we obtain that

$$
\alpha=\frac{1+\left(\beta \gamma \gamma_{3}\right) r}{1+\left(\gamma_{3}+s \beta \gamma \gamma_{1}-s \gamma_{1}\right) r} .
$$

By Sublemma $1, \alpha=1$, so that

$$
\beta \gamma \gamma_{3}=\gamma_{3}+s \beta \gamma \gamma_{1}-s \gamma_{1}
$$

or

$$
(\beta \gamma-1)\left(\gamma_{3}-s \gamma_{1}\right)=0 \text {. }
$$

Since either $\gamma_{1}$ and $\gamma_{3}$ is not 0 , it follows that $\beta \gamma-1=0$. Hence

$$
\gamma=1, \beta=1, \alpha=1 \text {. }
$$

If $\gamma_{2}$ is not 0 , we have

$$
\beta=\frac{1+\left(\alpha \gamma_{2}\right) s}{1+\left(\gamma \gamma_{2}+r \alpha \gamma_{1}-r \gamma \gamma_{1}\right) s} .
$$

Similarly,

$$
\gamma=1, \alpha=1, \beta=1 \text {. }
$$

Since $v=e_{2}$ and $w=e_{3}$, it follows that

$$
\Phi(v) w=\Phi\left(e_{2}\right) e_{3}=\Psi\left(e_{3}\right) e_{2}=\Psi(w) v .
$$

Hence the proof of Lemma 6 is completed.

Theorem 3. Let $\mathbf{K}$ be a division algebra of dimension $n, n=4$ or 8 , and let

$$
\pi: S^{2 n-1} \rightarrow \Sigma^{n}
$$

be the smooth great $(n-1)$-sphere fibration determined by $\mathbf{K}$ as seen in Theorem

2. Then $\mathbf{K}$ can be recovered from the fibration by the construction given above.

Proof. Let

$$
F_{0}=\{0\} \times S^{n-1}, \quad F_{1}=S^{n-1} \times\{0\},
$$


and let $\Sigma^{n}$ be assigned a Riemannian metric such that the smooth imbedding $\rho$ of $D^{n}=\{x \in \mathbf{K}|| x \mid \leqslant 1\}$ into $\Sigma^{n}$ given by

$$
\rho(v)=\pi\left(v w / \sqrt{|v w|^{2}+1}, w / \sqrt{|v w|^{2}+1}\right)
$$

is isometric. Then we have natural linear diffeomorphisms of $F_{0}, F_{1}$ and $S$ onto $S^{n-1}$, of which the first two are projections and the last is $(d \rho)^{-1}$.

Let us use these diffeomorphisms to identify $F_{0}, F_{1}$ and $S$ with $S^{n-1}$. Then $\xi: S \times F_{0} \rightarrow F_{1}$ becomes

$$
\xi: S^{n-1} \times S^{n-1} \rightarrow S^{n-1}
$$

defined by

$$
\xi(v, w)=v w /|v w| .
$$

Hence the regular multiplication constructed in Lemma 6 is the same as that in $\mathbf{K}$.

Theorem 4. Let

$$
\pi: S^{2 n-1} \rightarrow \Sigma^{n}
$$

be a given smooth great ( $n-1)$-sphere fibration, $n=4$ or 8 , and let $\mathbf{K}$ be the $n$-dimensional division algebra constructed from the fibration as seen earlier. Then the fibration is smoothly isomorphic to that determined by $\mathbf{K}$.

Proof. With respect to a preassigned Riemannian metric on $\Sigma^{n}$, there is a $\delta>0$ such that if $D_{\delta}$ is the closed $n$-disk in the tangent space of $\Sigma^{n}$ at $x_{0}$ of center 0 and radius $\delta$, then the exponential map exp imbeds $D_{\delta}$ smoothly into $\Sigma^{n}-\left\{x_{1}\right\}$. Let $D$ be the compact smooth $n$-manifold obtained from the disjoint union of $\Sigma^{n}-\left\{x_{0}\right\}$ and $S \times[0, \delta)$ by identifying every $(v, t) \in S \times$ $(0, \delta)$ with $\exp t v \in \Sigma^{n} \times\left\{x_{0}\right\}$. It is clear that $D$ is a smooth closed $n$-disk, and its boundary is $S \times\{0\}=S$.

Let

$$
\lambda: D_{\delta} \times F_{0} \rightarrow S^{2 n-1}
$$

be the smooth imbedding such that $\lambda(v, w) \in F_{1} w$ and $\pi \lambda(v, w)=\exp v$ for any $(v, w) \in D_{\delta} \times F_{0}$. Then we have a compact smooth $(2 n-1)$-manifold $W$ obtained from the disjoint union of $S^{2 n-1}-F_{0}$ and $S \times[0, \delta) \times F_{0}$ by identifying every $(v, t, w) \in S \times(0, \delta) \times F_{0}$ with $\lambda(t v, w) \in S^{2 n-1}-F_{0}$. It is clear that the boundary of $W$ is $S \times\{0\} \times F_{0}=S \times F_{0}$, and that $\pi: S^{2 n-1}-$ $F_{0} \rightarrow \Sigma^{n}-\left\{x_{0}\right\}$ can be naturally extended to a smooth fibration

$$
\pi: W \rightarrow D \text {. }
$$

From the construction of $\xi: S \times F_{0} \rightarrow F_{1}$, it can be shown that $\xi$ can be naturally extended to a smooth fibration

$$
\xi: W \rightarrow F_{1}
$$


such that for any $x \in W-\left(S \times F_{0}\right), \xi(x)$ is the point of intersection of $F_{0} x$ with $F_{1}$. Hence

$$
h_{1}=(\xi \times \pi)^{-1}: F_{1} \times D \rightarrow W
$$

is a diffeomorphism.

The inclusion map of $S^{2 n-1}-F_{0}$ into $S^{2 n-1}$ can be extended to a smooth map

$$
h_{2}: W \rightarrow S^{2 n-1}
$$

such that $h_{2}(v, w)=w$ for any $(v, w) \in S \times F_{0}=\partial W$. Therefore we have a smooth map

$$
h=h_{2} h_{1}: F_{1} \times D \rightarrow S^{2 n-1}
$$

such that the fibration $\pi: S^{2 n-1} \rightarrow \Sigma^{n}$ is induced by the projection fibration $F_{1} \times D \rightarrow D$. Moreover, whenever $(u, v),\left(u^{\prime}, v^{\prime}\right) \in F_{1} \times D, h(u, v)=h\left(u^{\prime}, v^{\prime}\right)$ iff either $(u, v)=\left(u^{\prime}, v^{\prime}\right)$ or $u=u^{\prime}, v, v^{\prime} \in S=\partial D$ and for some $w, w^{\prime} \in F_{0}$. $u=\xi(v, w)=\xi\left(v^{\prime}, w^{\prime}\right)=u^{\prime}$.

In the construction of the division algebra $\mathbf{K}$, we identify $F_{0}, F_{1}$ and $S$ with $S^{n-1} \subset \mathbf{K}$. Then we have a smooth map

$$
h^{\prime}: F_{1} \times D \rightarrow S^{2 n-1}
$$

given as follows. Let us regard $D-\left\{x_{1}\right\}$ as $\{v \in \mathbf{K}|0<| v \mid \leqslant 1\}$. Then for any $(u, v) \in F_{1} \times\left(D-\left\{x_{1}\right\}\right)$ there is a unique $w(u, v) \in \mathbf{K}$ with $v w(u, v)=u$. The map $h^{\prime}$ is given by

$$
h^{\prime}(u, v)=\left\{\begin{array}{l}
(u, 0) \quad \text { if } v=x_{1}, \\
\frac{u}{\sqrt{1+|w(u, v)|^{2}}}, \frac{w(u, v)}{\sqrt{1+|w(u, v)|^{2}}} \quad \text { otherwise. }
\end{array}\right.
$$

Now it is not hard to see that the identity map of $F_{1} \times D$ induces a smooth isomorphism between the fibration $\pi: S^{2 n-1} \rightarrow \Sigma^{n}$ and that determined by the division algebra $\mathbf{K}$.

Corollary 4. Up to a smooth isomorphism, every smooth great $(n-1)$-sphere fibration of $S^{2 n-1}$ is determined by an $n$-dimensional division algebra.

Remark. It is possible to have many $n$-dimensional division algebras, not isomorphic to one another but determining isomorphic smooth great $(n-1)$ sphere fibrations of $S^{2 n-1}$. In fact, whenever $\alpha, \beta, \gamma, \alpha^{\prime}, \beta^{\prime}, \gamma^{\prime}$ are positive real numbers satisfying

$$
\alpha+\beta+\gamma-\alpha \beta \gamma=\alpha^{\prime}+\beta^{\prime}+\gamma^{\prime}-\alpha^{\prime} \beta^{\prime} \gamma^{\prime},
$$


there is a 4-dimensional division algebra which, as the quaternionic algebra, has $\{e, i, j, k\}$ as a basis, but in which the multiplication is given by:

\begin{tabular}{c|cccc} 
& $e$ & $i$ & $j$ & $k$ \\
\hline$e$ & $e$ & $i$ & $j$ & $k$ \\
$i$ & $i$ & $-e$ & $\gamma k$ & $-\beta^{\prime} k$ \\
$j$ & $j$ & $-\gamma^{\prime} k$ & $-e$ & $\alpha i$ \\
$k$ & $k$ & $\beta j$ & $-\alpha^{\prime} i$ & $-e$
\end{tabular}

Also for any $\theta \in[0, \pi / 2]$, there is a 4-dimensional division algebra which has $\{e, i, j, k\}$ as a basis and in which the multiplication is given by:

\begin{tabular}{c|cccc} 
& $e$ & $i$ & $j$ & $k$ \\
\hline$e$ & $e$ & $i$ & $j$ & $k$ \\
$i$ & $i$ & $-e$ & $k$ & $-j$ \\
$j$ & $j$ & $-k$ & $-e \cos \theta+i \sin \theta$ & $i \cos \theta+e \sin \theta$ \\
$k$ & $k$ & $j$ & $-i \cos \theta-e \sin \theta$ & $-e \cos \theta+i \sin \theta$
\end{tabular}

For details, see Bruck [4]. Since all these division algebras are homotopic to the quaternionic algebra, the smooth great 3-sphere fibrations of $S^{7}$ determined by them are smoothly isomorphic to the Hopf fibration.

\section{References}

[1] J. F. Adams, On the non-existence of elements of Hopf invariant one, Ann. of Math. 72 (1960) 20-104.

[2] A. Beese, Manifolds all of whose geodesics are closed, Ergebnisse Math. und ihrer Grenzgebiete, Vol. 93, Springer, Berlin, 1978.

[3] A. Hurwitz, Über der Komposition der Quadratischen Form vom beliebig vielen Variablen, Nachr. Gesellsch. Wiss. Göttingen, 1898, 309-306; Math. Werke, Bd. II, 565-571.

[4] R. H. Bruck, Some results in the theory of linear non-associative algebras, Trans. Amer. Math. Soc. 56 (1944) 141-199.

University of Pennsylvania 
